\title{
MODELAGEM EM SÉRIES TEMPORAIS APLICADOS A NÚMEROS DE NOTIFICAÇÕES MENSAIS DE DENGUE EM PERNAMBUCO
}

\section{MODELING IN TEMPORARY SERIES APPLIED TO DENGUE MONTHLY NOTIFICATIONS NUMBERS IN PERNAMBUCO}

\author{
Jucarlos Rufino de Freitas ${ }^{1}$; André Luiz Pinto dos Santos ${ }^{2}$; Victor Casimiro Piscoya ${ }^{3}$; Ana \\ Luíza Xavier Cunha ${ }^{4}$; Moarcy Cunha Filho ${ }^{5}$
}

DOI: https://doi.org/10.31692/978-65-991061-7-0.258-269

\section{RESUMO}

Os casos de notificações de dengue (Aedes aegypti) no Brasil foram mencionados a partir de 1923. Desde então, até o início da década de 1980, tal vetor seria erradicado tanto no país como em outros países da América. Recentemente, está presente no Brasil em todos os 27 estados da Federação. Em 2017, segundo o Programa de Vigilância das Arboviroses da Secretaria Estadual de Saúde (SES), o Estado de Pernambuco informou que entre os 184 municípios, 156 (84,7\%) estão em risco de surto. Em relação aos anos anteriores, houve uma queda no número de pessoas adoecendo pelo arbovírus, mas devemos ressaltar que a arbovírus não foi riscado do mapa. O objetivo da pesquisa consiste em propor modelos estatísticos de Séries Temporais (ST) capazes de predizer possíveis surtos com base nas defasagens anteriores. Foram utilizados os dados mensais do Banco de Dados Meteorológicos para Ensino e Pesquisa (BDMEP), disponibilizados pelo Instituto Nacional de Meteorologia (INMET), no período de janeiro de 2000 a dezembro de 2016. A metodologia de Box \& Jenkins foi utilizada no estudo, isto é, os modelos SARIMA. A partir dos modelos propostos foram feitas as predições e a análise residual. Avaliando os resíduos pelo ajuste dos modelos, verificou-se que os resíduos não se comportam como uma sequência de variáveis aleatórias independentes, identicamente distribuídas com média zero e variância constante. Ainda, estes modelos conseguem captar o nível e inclinação (valores centrais), mas não as flutuações, ou seja, tais modelos são bons em predizer as observações para longos intervalos de tempo. Conclui-se que o modelo SARIMA apresenta melhores resultados (AIC), uma vez que consegue captar os comportamentos sazonais, além de apresentar com boa acurácia os casos de dengue de tais municípios. Portanto, esses modelos são boas ferramentas para profissionais de saúde que atuam na vigilância epidemiológica e carecem de informações que busquem fazer um planejamento de políticas de saúde e intervenções.

Palavras-Chave: Aedes aegypti. Séries Temporais, Predição.

\section{ABSTRACT}

The cases of notification of dengue (Aedes aegypti) in Brazil were mentioned from 1923. From that time until the beginning of the 1980s, such a vector would be eradicated both in the country and in other countries of America. Recently, it is present in Brazil in all 27 states of the Federation. In 2017, according to the Program of Surveillance of Arboviroses of the State Secretariat of Health (SES), the State of Pernambuco reported that among the 184 municipalities, $156(84.7 \%)$ are at risk of an outbreak. In relation to the previous years, there was a decrease in the number of people suffering from the arbovirus, but we must emphasize

\footnotetext{
${ }^{1}$ Pós Graduação em Biometria e Estatística Aplicada, UFRPE, jucarlos123@ hotmail.com

${ }^{2}$ Pós Graduação em Biometria e Estatística Aplicada, UFRPE, andredefensor@ hotmail.com

${ }^{3}$ Doutor, UFRPE, victor.piscoya@ufrpe.br

${ }^{4}$ Pós Graduação em Engenharia Ambiental, UFRPE, analuizaxcunha@gmail.com

${ }^{5}$ Doutor, UFRPE, moacyr.cunhafo@ufrpe.br
} 
that the arbovirus was not scratched from the map. The objective of the research is to propose statistical models of Time Series (ST) capable of predicting possible outbreaks based on previous lags. The monthly data from the Meteorological Database for Teaching and Research (BDMEP), made available by the National Institute of Meteorology (INMET), were used from January 2000 to December 2016. The Box \& Jenkins methodology was used in the study, ie the SARIMA models. Predictions and residual analysis were made from the proposed models. Evaluating the residuals by fitting the models, it was verified that the residuals do not behave as a sequence of independent random variables, identically distributed with zero mean and constant variance. Also, these models can capture the level and slope (central values), but not the fluctuations, that is, such models are good at predicting the observations for long intervals of time. It is concluded that the SARIMA model presents better results (AIC), since it manages to capture the seasonal behaviors, besides presenting with a good accuracy the cases of dengue of such municipalities. Therefore, these models are good tools for health professionals who act in epidemiological surveillance and lack information that seeks to plan health policies and interventions.

Keywords: Aedes aegypti. Time Series, Prediction.

\section{INTRODUÇÃO}

A dengue é uma doença infecciosa que, propagada por um arbovírus da família dos flaviviridae e transmitida ao homem através da picada de um mosquito (Aedes aegypti) e que inclui quatro sorotipos: DEN-1, DEN-2, DEN-3 e DEN-4 (DIAZ, 2004; ROSS, 2010). Atualmente, a dengue é um dos principais problemas de saúde pública do Brasil e do mundo.

Aedes aegypti é o vetor clássico do vírus da dengue e da febre amarela urbana. Este é um mosquito de hábito diurno e doméstico, utilizando-se preferencialmente de depósitos de água limpa para deposição dos ovos, os quais têm uma alta capacidade de resistir à dessecação. Essa característica tem revelado grande capacidade de adaptação a diferentes situações ambientais desfavoráveis (WHO, 2002).

O primeiro registro de casos de dengue no Brasil ocorreu na década de 1920. Durante os anos seguintes, não foram relatados casos no país, porém, por volta de 1970 à dengue retornou ao Brasil sendo responsável pela transmissão do vírus da dengue em epidemias registradas desde o início dos anos de 1980 achando-se disseminado em todos os 27 estados da federação (LOURENÇO-DE-OLIVEIRA et al., 2004).

Desde 1985, campanhas educativas institucionais vêm sendo realizadas pelas agências de saúde, com o objetivo de informar a população sobre doenças, combate, vetores e medidas preventivas. Estes se concentram nos períodos mais chuvosos, quando ocorrem os maiores níveis de infestação de vetores, e envolveram todos os meios disponíveis de acesso à comunidade (TAUIL, 2002). De acordo com os dados da literatura, as alternativas mais utilizadas para o controle da dengue no Brasil são métodos químicos, no entanto, existem outros métodos, como físicos, biológicos e genéticos (KOKOZA et al., 2000; PENNA, 2003). 
Não é fácil reconhecer um mosquito da dengue. A principal diferença para os demais pernilongos é a coloração escura e pequenas manchas brancas pelo corpo com duas listras em forma de lira no tórax. E é menor que um pernilongo. Prefere-se reproduzir na água limpa, estagnada, facilmente encontrada em ambientes doméstico e peridoméstico (TAUIL, 2001) ou descartáveis que acumulam água de chuvas, comumente encontrados nos lixos das cidades.

Diante do exposto, este trabalho consiste em aplicar modelos de Séries Temporais (ST) aos totais mensais do número de notificações de caso de dengue nos municípios de Serra Talhada, Ipojuca, Vitoria de Santo Antão, Recife e Petrolina, no período de 2000 a 2016, a fim de encontrar o modelo que melhor representa seu caráter preditivo para os próximos seis anos.

\section{FUNDAMENTAÇÃO TEÓRICA}

Segundo alguns autores (SILAWAN, 2008; MORETTIN, 2011; EARNEST et al. 2012; WONGKOON et al. 2012, SOEBIYANTO, 2010), modelos matemáticos e estatísticos, mas especificamente as ferramentas de análise de séries temporais, têm sido amplamente utilizados para monitorar e prever a incidência de dengue e outras doenças infecciosas. Em populações específicas, esses modelos contribuíram para o entendimento da dinâmica da doença, permitindo predizer o número de casos em períodos subsequentes às séries estudadas. Entre esses modelos, destaca-se o uso da SARISMA (Média Móvel Integrada Autorregressiva Sazonal), útil em situações em que os dados de séries temporais exibem flutuações sazonais periódicas que se repetem com aproximadamente a mesma intensidade a cada ano.

Por outro lado, a análise da tendência do crescimento (ou decrescimento) do número de notificações mensais de dengue para os cinco municípios foram obtidas através do uso da técnica de ST. A ST é um conjunto de observações ordenadas no tempo, não necessariamente igualmente espaçadas, a fim de verificar a dependência serial.

Desta forma, dizemos que uma série $Z=\left(Z_{1}, Z_{2}, Z_{3}, \cdots, Z_{t}\right)$ é fracamente estacionaria se sua média e variância forem constantes ao longo do tempo e o valor da covariância entre os dois períodos de tempo depender apenas da distância (GUJARATI, 2011). Na prática, as maiorias das ST apresentam algum tipo de não estacionariedade, sendo necessário aplicar diferenciações sucessivas para torna - lá estacionária. Normalmente, será suficiente tomar uma ou duas diferenças para que a série se torne estacionária.

Teste de Estacionariedade:

Ao realizar um teste de hipóteses, primeiramente definem-se as hipóteses nula $\left(H_{o}\right)$ e a alternativa $\left(H_{1}\right)$, depois se fixa o nível de significância do teste $(\alpha)$ em 0,05 . 


\section{Hipóteses:}

$\mathrm{H}_{0}: \phi=1, \mathrm{Z}_{\mathrm{t}}$ não é estacionária

$\mathrm{H}_{1}:|\phi|<1, \mathrm{Z}_{\mathrm{t}}$ é estacionária.

A verificação da estacionariedade foi realizada utilizando o teste Dickey-Fuller (DF), criado para verificar se o modelo auto-regressivo tem ou não raiz unitária. O teste estima a seguinte auto-regressão $\Delta Z_{t}=(\phi-1) Z_{t-1}+\mathrm{a}_{\mathrm{t}}$, em que $\mathrm{a}_{\mathrm{t}}$ é o choque aleatório com distribuição normal com média zero e variância $\left(\sigma^{2} a_{t}\right)$, e $\phi$ é o parâmetro associado a sua defasagem para o processo auto-regressivo.

Estatística do teste $(T)$ :

$T=\widehat{\phi} / S_{\widehat{\phi}} ;$

pvalor $=\left[Z>\left[T \mid H_{0}\right]\right]$

em que $\widehat{\phi}$ é o estimador do parâmetro associado a sua defasagem para o processo autoregressivo, $S$ é o desvio padrão dos parâmetros $\widehat{\phi}$, e $Z$ é o parâmetro da distribuição normal.

Regra de decisão: Se pvalor $<0,05$ rejeita $H_{0}$.

Teste para Tendência:

A tendência na ST representa o comportamento da série ao longo do tempo t, refletindo na evolução global no sentido do crescimento ou decrescimento do nível da série.

Hipóteses:

$\mathrm{H}_{0}$ : As observações da série não possuem tendências

$\mathrm{H}_{1}$ : As observações da série possuem tendência monotônica no tempo

Seja $y_{1}, y_{2}, y_{3}, \cdots, y_{n}$ as observações de uma série temporal. A partir do teste de Mann-Kendall (MK), foi analisado se a série é independente e identicamente distribuída. Sendo assim, sob $\mathrm{H}_{0}$ a estatística do teste $(S)$ é dada por

$S=\sum_{k=1}^{n-1} \sum_{j=k+1}^{n} \sigma\left(y_{j}-y_{k}\right)$ em que

$\sigma(y)=\left\{\begin{array}{c}1, y>0 \\ 0, \quad y=0 \\ -1, y<0\end{array}\right.$

Modelo Sazonal auto-regressivos integrado de Médias Móveis $\left(\operatorname{SARIMA}(\mathrm{p}, \mathrm{d}, \mathrm{q})(\mathrm{P}, \mathrm{D}, \mathrm{Q}){ }^{12}\right)$ :

Os modelos SARIMA(p,d,q)(P,D,Q) ${ }^{12}$ são modelos matemáticos não estacionário que apresentam uma periodicidade cíclica, com oscilações no decorrer da sua evolução temporal. Além, de captar o comportamento da autocorrelação entre os valores da ST $\left(\mathrm{Z}_{\mathrm{t}}\right)$, e com base nesse comportamento realizar previsões futuras. Os modelos $\operatorname{SARIMA}(\mathrm{p}, \mathrm{d}, \mathrm{q})(\mathrm{P}, \mathrm{D}, \mathrm{Q})^{12}$ resultam da combinação da sazonalidade $(S=12)$ e de três componentes (ou filtros):

1) $\operatorname{AR}(p)$, a série $Z_{t}$ é expressa como uma soma linear e finita de valores prévios do processo 
$Z_{t-1}, Z_{t-2}, \cdots, Z_{t-p}$ e um choque aleatório at. $\mathrm{O}$ modelo auto-regressivo é definida por $\mathrm{Z}_{\mathrm{t}}=$ $\phi_{1} \mathrm{Z}_{\mathrm{t}-1}+\phi_{2} \mathrm{Z}_{\mathrm{t}-2}+\cdots+\phi_{\mathrm{p}} \mathrm{Z}_{\mathrm{t}-\mathrm{p}}+\mathrm{a}_{\mathrm{t}}\left(\right.$ ou $\left.\phi(B) \mathrm{Z}_{\mathrm{t}}=\mathrm{a}_{\mathrm{t}}\right)$, em que $\phi_{\mathrm{i}}$ são os parâmetros da estrutura, $\mathrm{i}=1, \ldots, \mathrm{p}$ é a ordem da estrutura, $\mathrm{p}$ determina o número de passos entre as observações passadas e a previsão da observação seguinte e $\phi(B)=1-\phi_{1} \mathrm{~B}-\phi_{2} B^{2}-\cdots-$ $\phi_{\mathrm{p}} B^{2}$ representam os coeficientes do filtro $\operatorname{AR}(\mathrm{p})$.

2) I(d), a série é submetida à diferenciação com o intuito de torná-la estacionária (Box et al. 2015). A primeira diferença $Z_{t}$ é definida por $\Delta Z_{t}=Z_{t}-Z_{t-1}$, em que $Z_{t-1}$ é a defasagem anterior à $Z_{t}$. Analogamente a segunda diferença $\Delta^{2} Z_{t}$ é dada por $\Delta^{2} Z_{t}=Z_{t}-Z_{t-1}-$ $\left[Z_{t-1}-Z_{t-2}\right]=Z_{t}-2 Z_{t-1}+Z_{t-2}$, em que $Z_{t-2}$ é a defasagem anterior à $Z_{t-1}$.

3) MA(q), a série é expressa como a soma ponderada infinita de choques aleatórios $a_{t}$ ocorridos no período corrente e nos períodos passados (GUJARATI, 2011). O modelo media moveis é definido por $\mathrm{Z}_{\mathrm{t}}=\mathrm{a}_{\mathrm{t}}-\theta_{1} \mathrm{a}_{\mathrm{t}-1}-\theta_{2} \mathrm{a}_{\mathrm{t}-2}-\cdots-\theta_{\mathrm{q}} \mathrm{a}_{\mathrm{t}-\mathrm{q}}\left(\right.$ ou $\left.\mathrm{Z}_{\mathrm{t}}=\theta(B) \mathrm{a}_{\mathrm{t}}\right)$, em que $\theta_{\mathrm{i}}$ são parâmetros da estrutura, $\mathrm{i}=1, \ldots, \mathrm{q}$ é a ordem da estrutura e $\theta(B)=1-\theta_{1} \mathrm{~B}-$ $\left.\theta_{2} B^{2}-\cdots-\theta_{\mathrm{q}} B^{q}\right) \mathrm{a}_{\mathrm{t}}$ representam os coeficientes do filtro MA(q). Dessa forma, uma série pode ser modelada pelos três filtros ou apenas um subconjunto deles, resultando em vários modelos. A estrutura geral do modelo SARIMA(p,d,q)(P,D,Q) ${ }^{12}$ é definida por

$\phi(B) \Phi(B)^{S}\left(\Delta^{d} \Delta^{D} Z_{t}\right)=\theta(B) \Theta\left(B^{S}\right) a_{t}$

em que

$(i) \Phi(B)^{S}$ e $\Theta\left(B^{S}\right)$ representam os coeficientes sazonais do filtro auto-regressivo e do filtro médias móveis, respectivamente.

(ii) $\left(\Delta^{d} \Delta^{D} Z_{t}\right)$ é a série diferenciadas, com ordens d e $\mathrm{D}$, sendo $\mathrm{D}$ a ordem de diferenciação sazonal.

A ultima etapa a ser executada na modelagem $\operatorname{SARIMA}(\mathrm{p}, \mathrm{d}, \mathrm{q})(\mathrm{P}, \mathrm{D}, \mathrm{Q})^{12}$ consiste em fazer previsões de $\mathrm{h}$ passos à frente, a fim de obter a previsão do número de notificações mensais de dengue para os próximos seis anos. A previsão levou em consideração prever valores futuros e observar a tendência.

\section{METODOLOGIA}

Os dados utilizados no presente estudo se referem a número de notificações mensais de dengue em cinco municípios de Pernambuco (Serra Talhada, Ipojuca, Vitoria de Santo Antão, Recife e Petrolina) que foram disponibilizados pelo Instituto Nacional de Meteorologia (INMET), no período de janeiro de 2000 a dezembro de 2016.

Na base inicial de dados constavam os 185 municípios de Pernambuco, em que dois 
não puderam integrar a análise, pois para tais observações não foi possível ter acesso aos dados, inviabilizando a análise. Dos 183 municípios restantes, estávamos interessados em analisar o comportamento do número de notificações mensais de dengue para as cinco mesorregiões do estado de Pernambuco: Sertão (Serra Talhada), Agreste (Ipojuca), Zona da

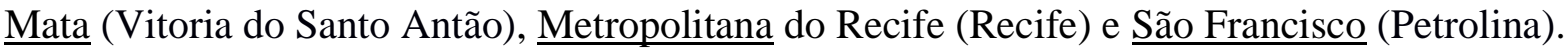

A fim de modelar o número de notificações mensais de dengue para os cinco municípios, foi proposta a análise de ST. Uma modelagem estatística capaz de quantificar e prever um determinado atributo ao longo do tempo, cujo objetivo consiste em elaborar um modelo estatístico que descreva adequadamente o comportamento de um determinado atributo ao longo do tempo, e maneira que as implicações teóricas do modelo sejam compatíveis com as amostras observadas nas ST. A partir do modelo ajustado a ST é possível prever a evolução futura da série ou explicar a relação entre os distintos componentes do modelo. Para a análise estatística dos dados, foram utilizados os softwares estatísticos RStudio e SPSS Statistics 20.

\section{RESULTADOS E DISCUSSÃO}

Como se podem observar as séries do número de notificações mensais de dengue para o município de Serra Talhada, Ipojuca Vitoria do Santo Antão, Petrolina, Figura 1a e Recife Figura 1b, no período de janeiro de 2000 a dezembro de 2016, não parecem adequar a distribuição Gaussiana e as observações apresentam autocorrelação.

Figura 1: Histogramas do número de notificações mensais de dengue para o município de Serra Talhada, Ipojuca, Vitoria do Santo Antão, Petrolina (a) e região metropolitana do Recife (b) no período de janeiro de 2000 a dezembro de 2016. Fonte: Própria.

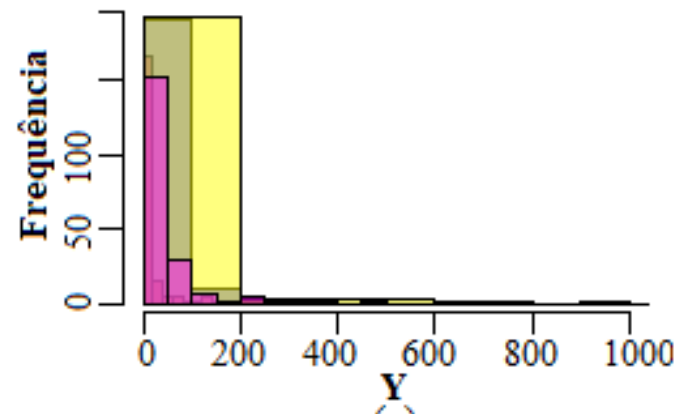

(a)

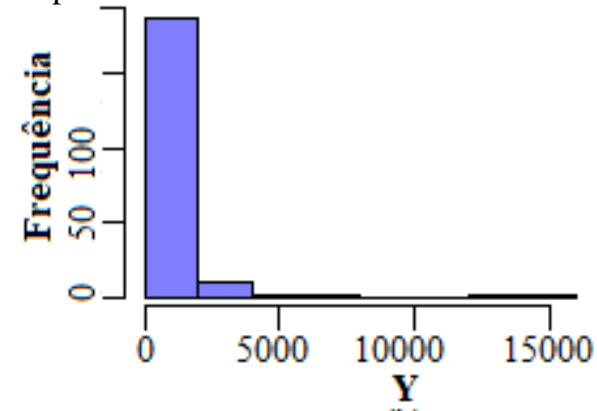

(b)

Deste modo, seja $Z=\left(Z_{1}, Z_{2}, Z_{3}, \cdots, Z_{t}\right)^{T}$ o vetor do número de notificações mensais de dengue em cinco municípios de Pernambuco no período de janeiro de 2000 a dezembro de 2016. Para a modelagem dos dados, se utilizou a transformação logaritmo natural $Z^{*}=$ $\left(\log \left(Z_{1}+1, Z_{2}+1, Z_{3}+1, \cdots, Z_{t}+1\right)\right)^{T}$, para minimizar os efeitos dos meses com grandes notificações. Em alguns casos não há como admitir valores $\log (0)$ e, portanto foi necessário incrementar uma unidade na série original. Cabe ressaltar que, essa abordagem não provocou 
mudanças expressivas na característica das séries, onde reduziu a grande variabilidade dos números de casos de dengue mensais (MARTINEZ; SILVA; FABBRO, 2011). As observações apresentam uma periodicidade sazonal de comprimento 12 (Figura 2), isso já era esperado, uma vez que a incidência de dengue apresenta um ciclo sazonal anual com maior incidência no verão do que no inverno. Segundo Vilches e Ferreira (2013), afirmam que a inclusão do efeito sazonal e de uma fase entre as taxas de reposição e de mortalidade do vetor podem explicar a periodicidade dos picos epidêmicos de dengue.

Figura 2: Resposta mensal do número de notificações de dengue para os municípios de Ipojuca e Serra Talhada (a), Vitoria do Santo Antão., Recife e Petrolina (b) no período de janeiro de 2000 a dezembro de 2016. Fonte: Própria.



(a)

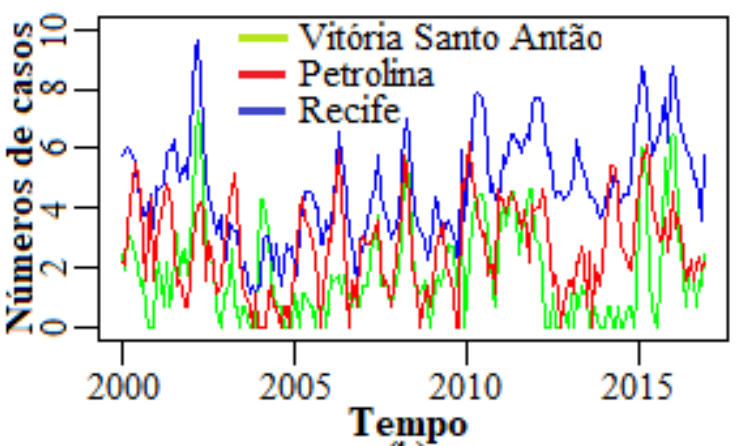

(b)

O comportamento das funções de autocorrelação (FAC) para os cinco municípios apresentam um decaimento exponencial senoidal com periodicidade sazonal de comprimento 12 (Figura 2), onde é observado um padrão temporal oscilatório em torno dos Lags.

Figura 3: Correlograma da função de autocorrelação (ACF) da série temporal aplicada a função logaritmo natural, para os cincos municípios de Pernambuco: Serra Talhada (a), Ipojuca (b), Vitoria do Santo Antão (c),



(a)
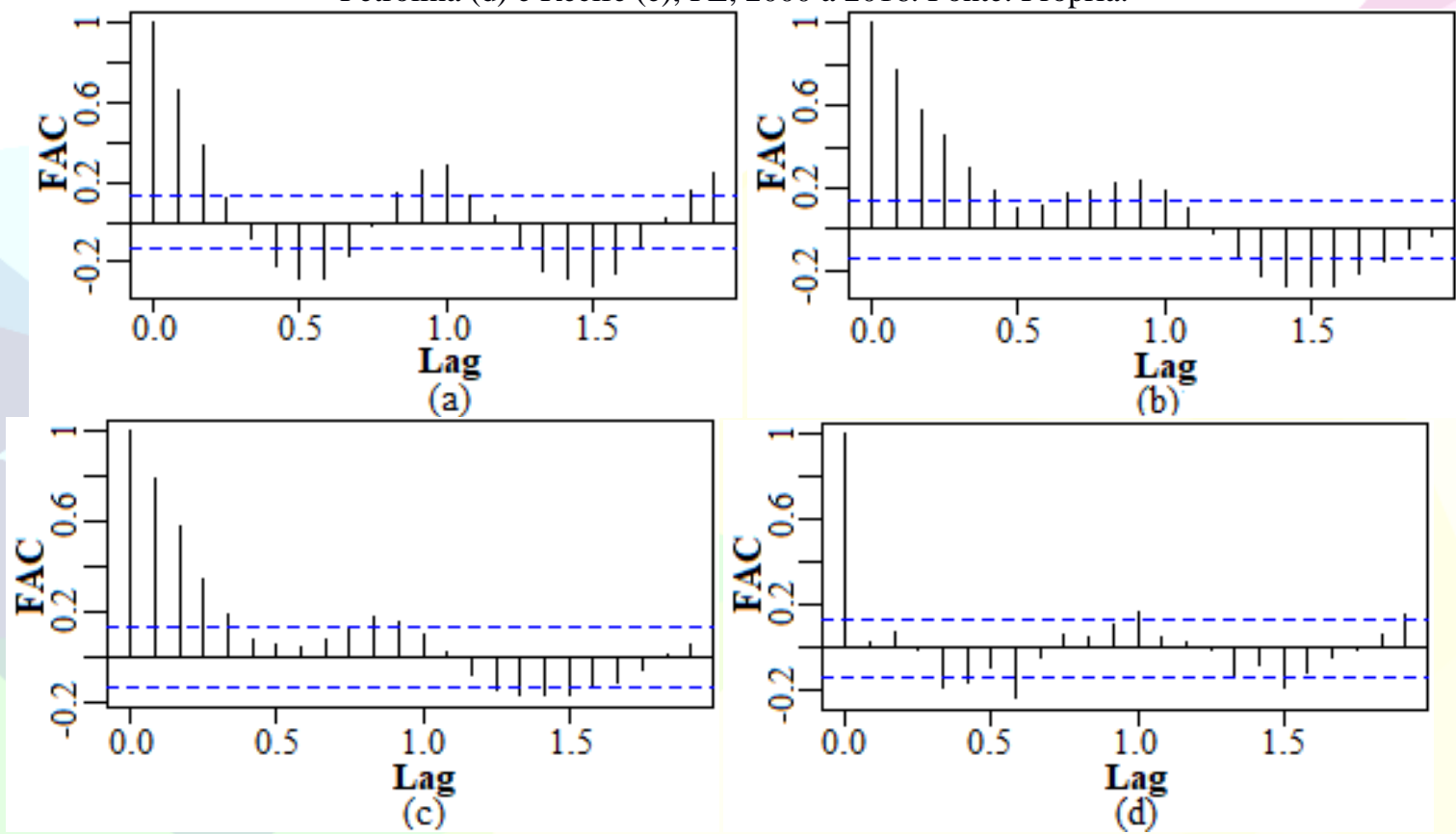

(b)

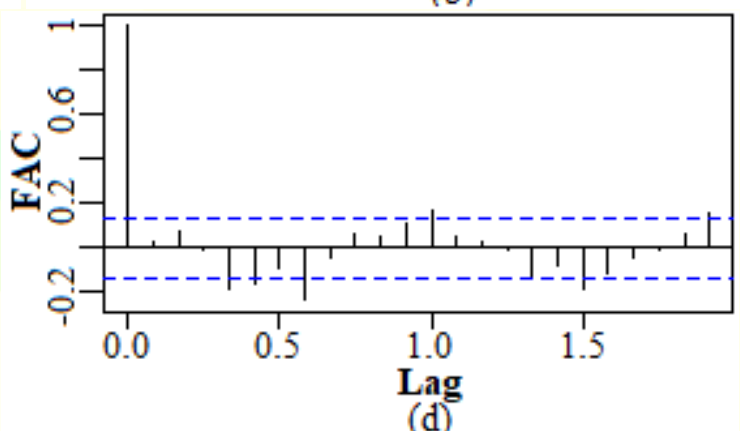




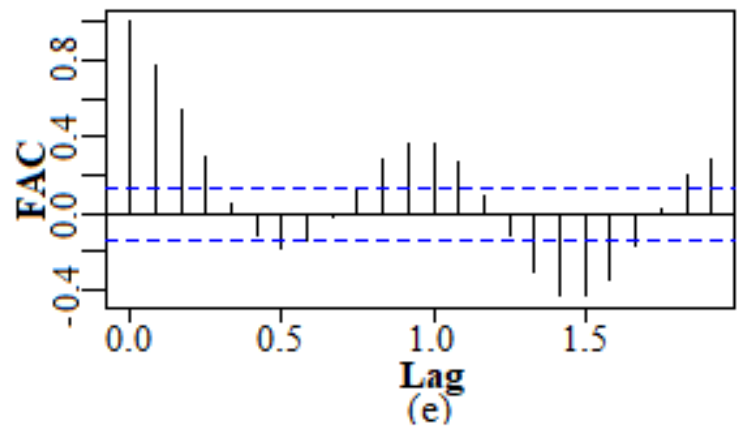

A Tabela 1 apresenta os resultados das análises de tendência e estacionariedade das ST, onde foram utilizados os testes de MK e DF, com nível de significância de 5\%. A partir dos resultados do teste de MK pode-se verificar que o município de Ipojuca e Recife apresentou uma tendência significativa ( $\mathrm{p}$-valor $<0,05$ ), sendo necessário aplicar a primeira diferença na série. Entretanto, pelo teste de DF, observa-se que a hipótese foi rejeitada para todos os municípios. Sendo assim, admite-se que todas as ST são estacionárias.

Tabela 1: Resultados obtidos com a aplicação dos respectivos testes, DF e MK, para os cinco municípios de Pernambuco no período de janeiro de 2000 a dezembro de 2016. Fonte: Própria.

\begin{tabular}{c|c|ccc|c|c}
\hline Municípios & Serra Talhada & Ipojuca & Vitória do santo Antão & Recife & Petrolina \\
\hline DF & $-6,35$ & $-4,91$ & $-4,08$ & $-3,59$ & $-5,53$ \\
p-valor & $<\mathbf{0 , 0 1}$ & $<\mathbf{0 , 0 1}$ & $<\mathbf{0 , 0 1}$ & $<\mathbf{0 , 0 1}$ & $<\mathbf{0 , 0 1}$ \\
MK & 0,073 & 0,161 & 0,016 & 0,262 & 0,081 \\
p-valor & 0,136 & $<\mathbf{0 , 0 1}$ & 0,743 & $<\mathbf{0 , 0 1}$ & 0,088 \\
\hline
\end{tabular}

Após a aplicação dos testes de DF e MK, segue-se com o ajuste dos modelos para os cinco municípios, os quais se podem observar na Tabela 2. Podendo concluir que os modelos, $\operatorname{ARIMA}(\mathrm{p}, \mathrm{d}, \mathrm{q})_{12}$ e $\operatorname{SARIMA}(\mathrm{p}, \mathrm{d}, \mathrm{q})(\mathrm{P}, \mathrm{D}, \mathrm{Q})_{12}$, foram os que melhor se ajustaram aos dados através do critério AIC proposto por Schwarz (1978).

Tabela 2: Seleção dos melhores modelos ajustados considerando os valores do AIC para os cinco municípios de Pernambuco no período de janeiro de 2000 a dezembro de 2016. Fonte: Própria.

\begin{tabular}{c|c|c|c}
\hline Municípios & Modelo & $\widehat{\boldsymbol{\sigma}}^{\mathbf{2}} \boldsymbol{a}_{\boldsymbol{t}}$ & AIC \\
\hline Serra Talhada & SARIMA $(2,0,2)(1,0,0)_{12}$ & 1,008 & 590,39 \\
Ipojuca & ARIMA $(2,0,2)_{12}$ & 0,813 & 542,02 \\
Vitória do Santo Antão & ARIMA $(1,0,2)_{12}$ & 0,992 & 584,44 \\
Recife & SARIMA $(0,0,0)(0,0,2)_{12}$ & 0,698 & 508,08 \\
Petrolina & SARIMA $(2,0,3)(1,0,0)_{12}$ & 0,866 & 560,06 \\
\hline
\end{tabular}

Após a formulação e ajuste do modelo, foi realizada a análise dos resíduos para avaliar os pressupostos de adequação do modelo. Avaliando os resíduos (Figura 4), verificou-se que os resíduos não se comportam como uma sequência de variáveis aleatórias independentes, identicamente distribuídas com média zero e variância constante. Portanto, estes modelos conseguem captar o nível e inclinação (valores centrais), mas não as flutuações, ou seja, estes modelos são bons em predizer as observações em distantes intervalos de tempo. Martinez, Silva e Fabbro (2011), encontraram valores distintos, em relação aos resíduos, em que 
sugerem que os resíduos do modelo seguem distribuição normal.

Figura 4: Gráficos de análise de resíduo dos modelos SARIMA(p,d,q)(P,D,Q) 12 para os cinco municípios de

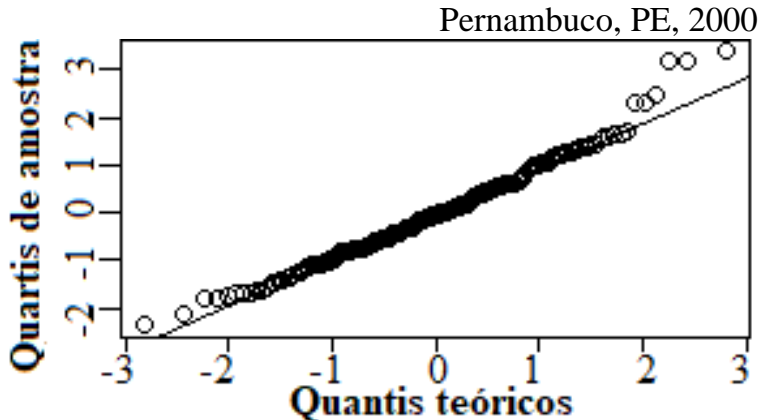

(a)
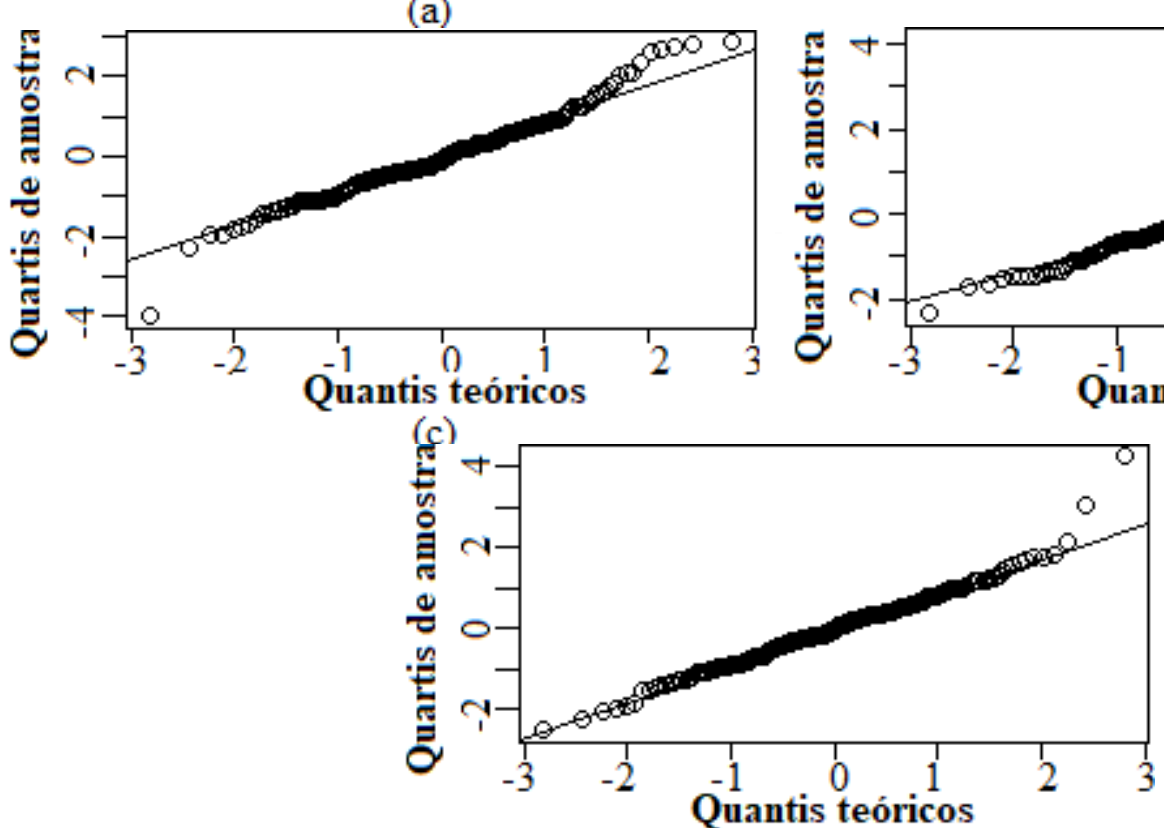

(e)

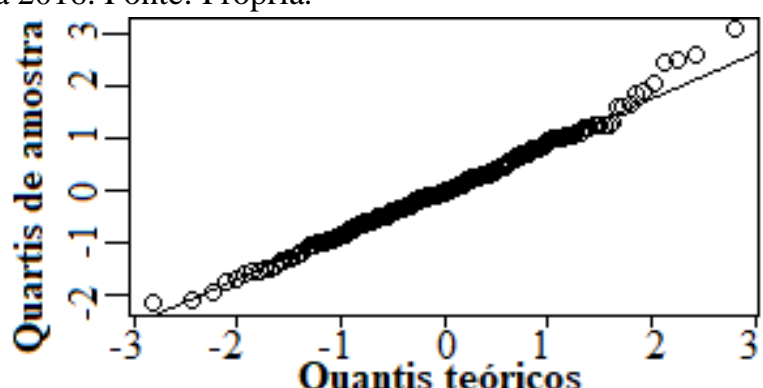

(b)

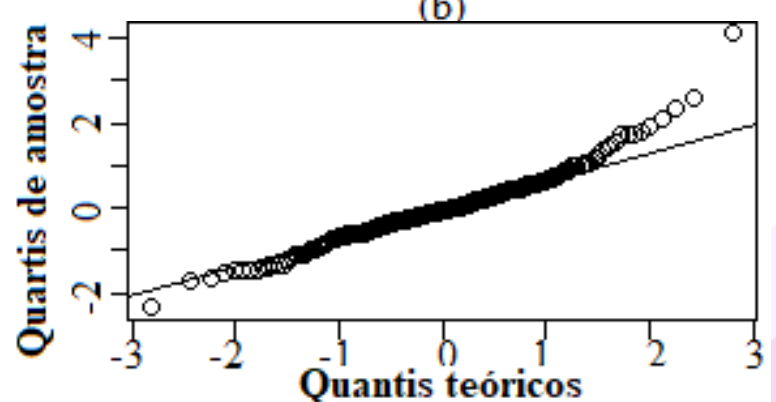

(d)

A partir dos modelos acima, se efetuou a predição dos meses de janeiro de 2017 a dezembro de 2022 (Figura 5). Com base nos valores preditos pelo modelo, a um nível de 95\% de confiança, espera-se que ocorram aproximadamente 64(a), 121(b), 1845(c), 81(d) e 178(e) notificações de dengue em 2018, o que indica uma redução em comparação ao ano de 2016. Para os municípios de (a), (d) e (e) o modelo estimado conseguiu captar o comportamento da série original. Já para os municípios (b) e (c) as estimativas apresentaram valores distintos do valor real, principalmente em Recife, pois foi aplicado uma diferenciação na série original para retirar o efeito da tendência, reduzindo assim, a variabilidade dos dados. A diminuição dos casos das arboviroses decorreu de diversos fatores, entre eles o empenho da sociedade na eliminação dos criadouros do mosquito Aedes aegypti. Vale ressaltar que o números de notificações são maiores períodos quentes e chuvosos (DE ANDRADE OLIVEIRA et al., 2018), principalmente no verão, quando o mosquito tem o clima ideal para se proliferar. 
Figura 5: Resultado da predição mensais do número de notificações de dengue obtida do ARIMA(p,d,q) e SARIMA(p,i,d)(P,I,D) para os municípios de Serra Talhada (a), Ipojuca (b), Recife (c), Vitoria do Santo Antão



(a)

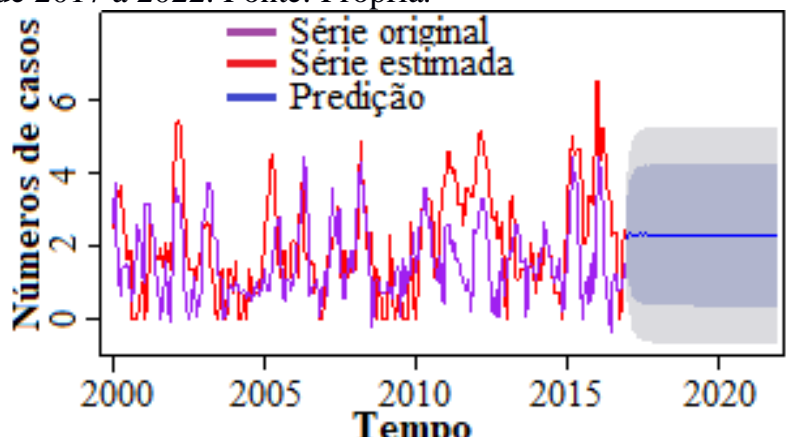

(b)

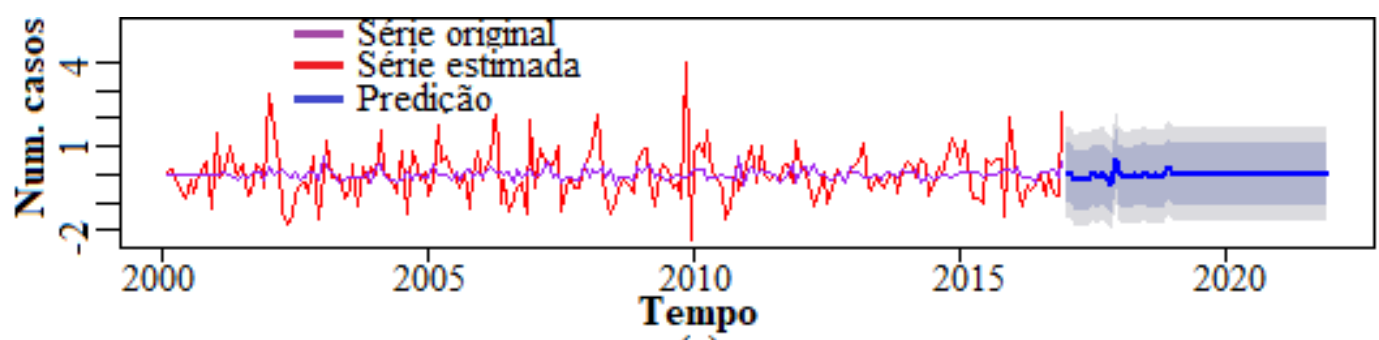

(c)

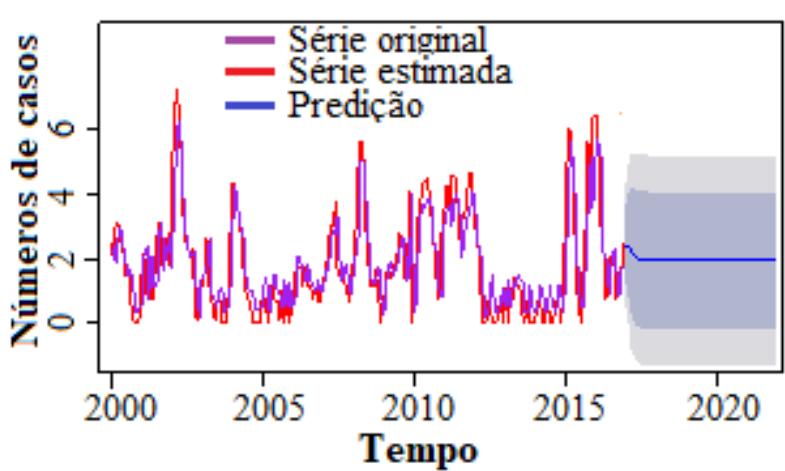

(d)

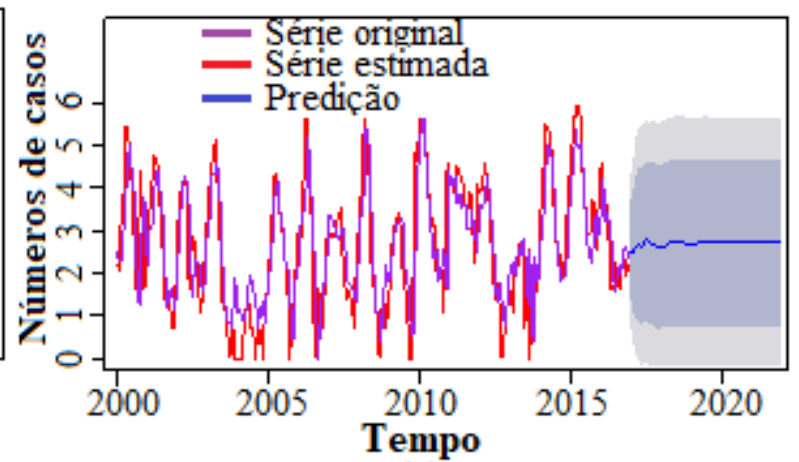

(e)

\section{CONCLUSÕES}

A dengue é uma doença transmitida por vetores, sendo o principal deles o Aedes aegypti. Portanto, a busca por relações entre fatores ambientais e a disseminação da doença tem sido investigada para estabelecer mecanismos de controle e prevenção de epidemias. que tem crescido nos últimos anos, o investimento em pesquisa para explicar o comportamento epidemiológico da doença.

Existem vários modelos estatísticos descritos na literatura para explicar a incidência da dengue. No entanto, existem divergências quanto à validade real de modelos baseados em fatores climáticos e modelos baseados em variáveis relacionadas ao controle vetorial, uma vez que a variabilidade apresentada por essas variáveis não é suficiente para explicar satisfatoriamente o comportamento estatístico da incidência de dengue.

$\mathrm{Na}$ nossa pesquisa, dos modelos estimados para os cincos municípios, o que apresentou os melhores resultados foi o SARIMA, uma vez que a incidência de dengue 
apresenta um ciclo sazonal anual com maior incidência no verão do que no inverno. Desse modo, os modelos SARIMA possuem a capacidade de fazer predição com boa acurácia dos números de notificações de dengue para tais municípios, por meio da apresentação de boas ferramentas para profissionais de saúde que trabalham na vigilância epidemiológica e carecem de informações que busquem fazer um planejamento de políticas de saúde e intervenções. Embora já exista uma vacina aprovada no Brasil e outras em desenvolvimento, práticas educativas nas escolas e nas comunidades são alternativas viáveis e de baixo custo na eliminação de criadouros.

\section{REFERÊNCIAS}

BOX, G. E.; JENKINS, G. M.; REINSEL, G. C.; LJUNG, G. M. (2015). Time series analysis: forecasting and control. John Wiley \& Sons.

DIAZ, J. F. (2004). Epidemiología molecular del dengue en las Americas. Memorias del primer simposio nacional de Virología, Universidad de Antioquia, Medellín, Colombia. Iatreia 17:7.

EARNEST, A.; TAN, S.B.; WILDER-SMITH, A.; MACHIN, D. (2012) Comparing statical models to predict dengue fever notifications. Computational and Mathematical Methods in Medicine.

GUJARATI, D. N.; PORTER, D. C. (2011). Econometria Básica-5. Amgh Editora.

DE ANDRADE OLIVEIRA, M. A. C., COELHO, F. A., DE BARROS FREITAS, R., TAVARES, A. P., SILVA, Í. N. D. P. N., DA SILVA PINTO, S.; ANDRADE, F. M. (2018). Perfil das notificações de dengue e sazonalidade no município de ubá-mg, 2015 a 2016. Revista Científica FAGOC-Saúde, 2(2), 9-14.

LOURENÇO-DE-OLIVEIRA, R.; VAZEILLE, M.; FILIPPIS, A. M. B.; FAILLOUX, A. B. (2004). Aedes aegypti in Brazil: genetically differentiated populations with high susceptibility to dengue and yellow fever viruses. Trans R Soc Trop Med Hyg 98:43-54.

MARTINEZ, E. Z., SILVA, E. A. S. D.; FABBRO, A. L. D. (2011). A SARIMA forecasting model to predict the number of cases of dengue in Campinas, State of São Paulo, Brazil. Revista da Sociedade Brasileira de Medicina Tropical, 44(4), 436-440.

MORETTIN, P. A. (2011). Econometria financeira: um curso em séries temporais financeiras. 2 a edição. São Paulo: Edgar Blucher.

ROSS, T. M. (2010). Dengue virus. Clinics in Laboratory Medicine, 30(1):149-60.

SILAWAN, T.; SINGHASIVANON, P.; KAEWKUNGWAL, J.; NIMMANITYA, S.; SUWONKERD, W. (2008). Temporal patterns and forecast of dengue infection in Notheastern Thailand. Southeast Asian. J Trop Med Public Health, v. 39, n. 1, p. 90-98. 
SOEBIYANTO, R. P.; ADIMI, F.; KIANG, R.K.(2010). Modeling and predicting seasonal influenza transmission in warm regions using climatological parameters. Plos One, v. 5, n. 3, p. $1-10$.

TAUIL, P. L. (2001). Urbanização e ecologia do dengue. Cadernos de Saúde Pública, 17, S99-S102.

VILCHES, T. N., \& FERREIRA, C. P. (2013). Um modelo para a dengue com influência sazonal. TEMA (São Carlos), 14(3), 279-290.

WHO - World Health Organization. Dengue prevention and control. Geneva: World Health Organization; 2002.

WONGKOON, S.; JAROENSUTASINEE, M.; JAROENSUTASINEE, K. (2012) Development of temporal modeling for prediction of dengue infection in Northeastern Thailand. Asian Pacific Journal of Tropical Medicine, v. 5, n. 3, p. 249-252. 